\title{
May chaos always be suppressed by parametric perturbations?
}

\author{
Tilo Schwalger \\ Department of Physics, Humboldt University, Berlin, Germany \\ Arsen Dzhanoev and Alexander Loskutov \\ Physics Faculty, Moscow State University, Moscow 119899, Russia
}

(Received 31 October 2005; accepted 23 March 2006; published online 11 May 2006)

\begin{abstract}
The problem of chaos suppression by parametric perturbations is considered. Despite the widespread opinion that chaotic behavior may be stabilized by perturbations of any system parameter, we construct a counterexample showing that this is not necessarily the case. In general, chaos suppression means that parametric perturbations should be applied within a set of parameters at which the system has a positive maximal Lyapunov exponent. Analyzing the known DuffingHolmes model by a Melnikov method, we showed that chaotic dynamics cannot be suppressed by harmonic perturbations of a certain parameter, independently from the other parameter values. Thus, to stabilize the behavior of chaotic systems, the perturbation and parameters should be carefully chosen. (0) 2006 American Institute of Physics. [DOI: 10.1063/1.2195787]
\end{abstract}

Great success and profit of the chaos suppression phenomenon in applications led to the widespread opinion that chaotic oscillations may always be stabilized by parametric perturbations. Nevertheless, in what cases can the chaos be suppressed in such a manner? In general, this question means that we should perturb the system within a region of parameter values where chaotic behavior occurs. The chaoticity region may be determined as a set of parameters for which the separatrices are split. In this case, the maximal Lyapunov exponent is always positive. Thus, the chaos suppression implies that all perturbed parameters should not fall outside the limits of this region. In the present paper, by a DuffingHolmes system we construct an analytic example when parametric perturbations cannot lead to the suppression of chaos if they belong to the chaoticity region. One can expect that the same results may be observed in the other physical systems. Our analysis is based on the Melnikov method, which gives us a criterion for the observation of chaos. The obtained results are in excellent agreement with numerical simulations.

\section{INTRODUCTION}

As is known, chaotic oscillations are inherent in many natural processes. But the development of chaos is sometimes not desirable, and thus it is necessary to create conditions under which originally chaotic systems acquire regular dynamics. In this connection, in the past 15 years, problems related to suppressing and controlling chaos have attracted much attention. Stabilization of chaotic oscillations may be realized in two ways. The first does not take into account the current value of the dynamical variables of the system, whereas the qualitatively different second method involves feedback as a necessary component of the system. By the established convention, the first method is called suppression of chaos and the second one is called (feedback) controlling chaos.
Using the feedback we have a certain advantage, because in most cases feedback control produces the required result: a prescribed saddle limit cycle is stabilized and the system thus attains the necessary motion. On the other hand, stabilization methods without feedback do not require continuous tracking of the system state and are more robust to noise.

The idea that chaos may be suppressed goes back to Refs. 1 and 2, where it was proposed to perturb periodically the system parameters. Later, this approach gained analytical substantiation in a series of publications ${ }^{3-9}$ (as a review, see Ref. 10). At the same time, it has been found that chaotic systems can be controlled. ${ }^{11-13}$

We will focus on chaos suppression. This phenomenon means the following. Let us suppose that for required values of parameters, the chaotic dynamics is not acceptable. Then, to realize periodic behavior and suppress chaos, it is sufficient to apply a weak parametric perturbation that does not fall outside the set of parameter values for which the system exhibits chaotic properties, e.g., one that has a positive Lyapunov exponent. Great successes of chaos suppression phenomena in applications led to the opinion that chaos can always be suppressed by such perturbations. However, this is not the case. Using a Duffing oscillator, we analytically construct a counterexample in which periodic perturbations cannot lead to the suppression of chaos. As a consequence, for an arbitrary system we do not know in advance what parameter is appropriate for the stabilization of the system dynamics.

The Duffing system is known as one of the simplest nonlinear dissipative models with a wide range of complex behavior. It is used for the description of many real processes, such as mechanical and radio physical oscillations (see, e.g., Refs. 14 and 15 and references therein), plasma dynamics (see Ref. 16), and others. The properties of the Duffing system have been widely analyzed. In particular, using the Melnikov method, ${ }^{14,17,18}$ which is based on the analy- 
sis of the homoclinical structure of stable and unstable manifolds of a hyperbolic point, it has been shown that at certain parameter values this equation has chaotic dynamics. ${ }^{14,21}$

Modifications of the Duffing oscillator have also attracted a considerable amount of interest as an appropriate model for the investigation of the chaos suppression phenomenon (see Refs. 3, 8, 19, and 20 and references cited therein). One of the modified Duffing equations is known as the Duffing-Holmes system:

$$
\ddot{x}+\delta \dot{x}-x+\beta(1+\eta \cos \Omega t) x^{3}=\gamma \cos \omega t .
$$

In the present paper, by means of the Melnikov method we show that periodic perturbations of the parameter $\delta$ do not lead to a stabilization of the chaotic behavior of the system (1) if these perturbations belong to the chaotic region. In the case of the nonlinear oscillatory circuit, perturbations of $\delta$ may be realized by temporal variations of the inductance.

\section{A. The Melnikov method}

It is well known that in Hamiltonian systems, separatrices can split. In this case, stable and unstable manifolds of a hyperbolic point do not coincide, but intersect with each other in an infinite number of homoclinical points. [Usually the motion in the $(n+1)$-dimensional phase space $\left(x_{1}, \ldots, x_{n}, t\right)$ is considered in the projection onto an $n$-dimensional hypersurface $t=$ const (Poincaré section).] The presence of such points gives us a criterion for the observation of chaos. This criterion can conveniently be obtained by the Melnikov function (MF), which "measures" (in the first order of a small perturbation parameter) the distance between stable and unstable manifolds. Let us consider a twodimensional autonomous system under the action of a periodic perturbation,

$$
\dot{\mathbf{x}}=\mathbf{f}_{0}(\mathbf{x})+\varepsilon \mathbf{f}_{1}(\mathbf{x}, t) .
$$

Let furthermore $\mathbf{x}_{0}$ be the separatrix of the unperturbed system $\dot{\mathbf{x}}=\mathbf{f}_{0}(\mathbf{x})$. Then the MF at any given time $t_{0}$ is defined as follows:

$$
D\left(t_{0}\right)=-\left.\int_{-\infty}^{+\infty} \mathbf{f}_{0} \wedge \mathbf{f}_{1}\right|_{\mathbf{x}=\mathbf{x}_{0}\left(t-t_{0}\right)} d t
$$

where the integral is taken along the unperturbed separatrix $\mathbf{x}_{0}\left(t-t_{0}\right)$ and the integrand is $\mathbf{f}_{0} \wedge \mathbf{f}_{1}=f_{0 x} f_{1 y}-f_{0 y} f_{1 x}$.

In general, in dissipative systems one can observe three possibilities for the MF: either $D\left(t_{0}\right)<0$ [Fig. 1(b)], $D\left(t_{0}\right)$ $>0$ [Fig. 1(c)] for any $t_{0}$, or $D\left(t_{0}\right)$ changes its sign for some $t_{0}$ [Fig. 1(d)]. Only in the last case does chaotic dynamics arise. Thus, the MF determines the character of the motion near the separatrix. Note that the Melnikov method has a perturbative character, thus its application is allowed only for trajectories that are sufficiently close to the unperturbed separatrix. Moreover, this method is adaptive only for systems with $\varepsilon \ll 1$.

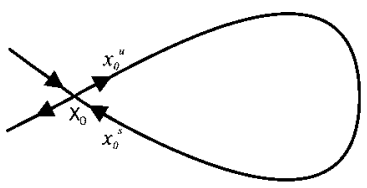

a)

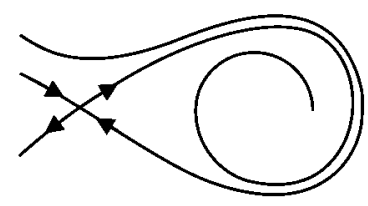

c)

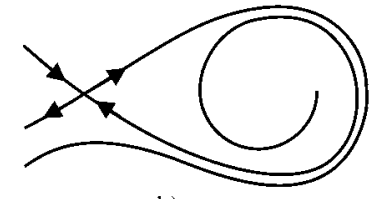

b)

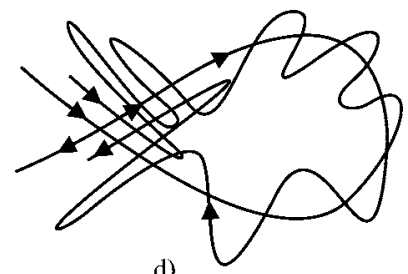

d)
FIG. 1. Poincaré section $t=$ const $(\bmod T)$ of the system (2) for $\varepsilon=0$ (a) and $\varepsilon \neq 0$ (b)-(d).

\section{CHAOTIC REGIONS IN THE DUFFING-HOLMES SYSTEM}

Let us consider the Duffing-Holmes equation in the following form:

$$
\dot{x}=y, \quad \dot{y}=x-\beta(1+\eta \cos \Omega t) x^{3}-\delta y+\gamma \cos \omega t .
$$

Following Eq. (2), $\mathbf{f}_{0}=\left(y, x-\beta x^{3}\right)$ and $\mathbf{f}_{1}=(0, \gamma \cos \omega t$ $-\beta \eta \cos (\Omega t) x^{3}-\delta y$ ). If parameters $\gamma, \eta$, and $\delta$ are small enough, we can apply the Melnikov method. The MF given by (3) yields

$$
\begin{aligned}
D\left(t_{0}\right)= & -\int_{-\infty}^{+\infty}\left[\gamma \cos \omega t-\beta \eta \cos (\Omega t) x_{0}^{3}\left(t-t_{0}\right)\right. \\
& \left.-\delta y_{0}\left(t-t_{0}\right)\right] y_{0}\left(t-t_{0}\right) d t .
\end{aligned}
$$

The explicit expressions for the unperturbed separatrix $\left(x_{0}(t), y_{0}(t)\right)$ are determined by the equation $H_{0}\left(x_{0}(t), y_{0}(t)\right)$ $=0$, where $H_{0}(x, y)=\frac{1}{2} y^{2}-\frac{1}{2} x^{2}+\beta / 4 x^{4}$ is the Hamiltonian of the unperturbed system. Thus, we obtain $x_{0}(t)=\kappa / \cosh t$ and $y_{0}(t)=-\kappa \sinh t / \cosh ^{2} t$ as a parametrization of the separatrix, where $\kappa=\sqrt{2 / \beta}$. Finally, the MF is ${ }^{22}$

$$
D\left(t_{0}\right)=-A \sin \omega t_{0}+B \sin \Omega t_{0}+C
$$

with the constants

$$
\begin{aligned}
& A=\pi \kappa \gamma \omega \operatorname{sech}(\pi \omega / 2), \\
& B=(1 / 24) \pi \beta \eta \kappa^{4} \Omega^{2}\left(4+\Omega^{2}\right) \operatorname{csch}(\pi \Omega / 2), \\
& C=(2 / 3) \kappa^{2} \delta .
\end{aligned}
$$

Let us now introduce the subset $\Delta_{c}$ of the parameter range $\delta$ such that if $\delta \in \Delta_{c}$, then the system (4) exhibits chaotic properties. To find this chaotic region, we have to examine under which values of $\delta$ the MF changes its sign. For the case $\omega=\Omega$, the analysis gives an explicit expression for $\Delta_{c}$,

$$
\delta<\delta_{\mathrm{cr}} \equiv \frac{3}{2 \kappa^{2}}|B-A| .
$$




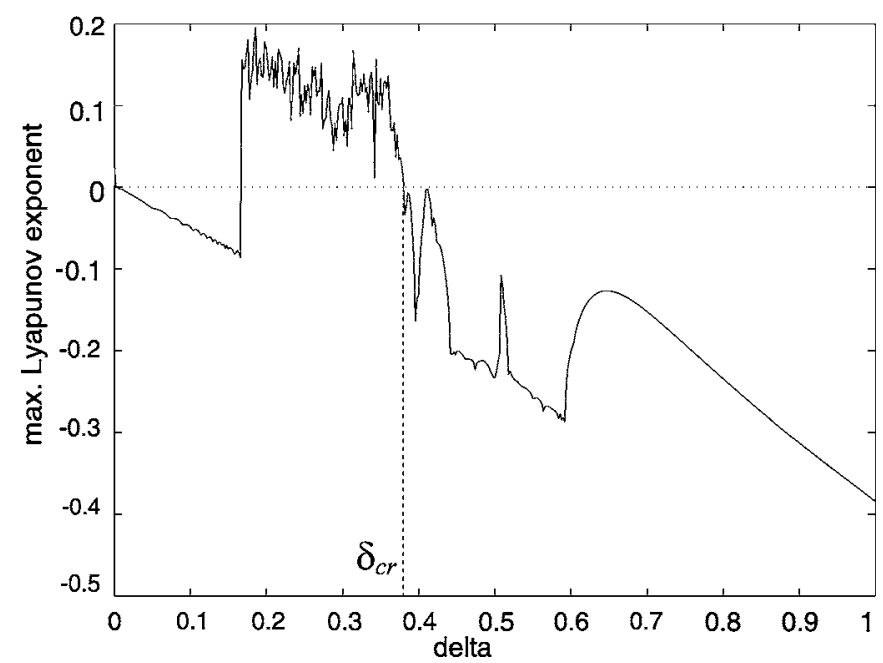

FIG. 2. The maximal Lyapunov exponent. The parameter values are the following: $\omega=\Omega=1.1, \gamma=0.114, \eta=0.03, \beta=8$. Initial conditions: $x_{0}=0$, $y_{0}=0$.

If $\omega \neq \Omega$, it has to be considered whether the frequencies $\omega$ and $\Omega$ are commensurable. When they are incommensurable, i.e., $\omega / \Omega$ is irrational, we immediately have

$$
\delta<\delta_{\mathrm{cr}} \equiv \frac{3}{2 \kappa^{2}}(A+B)
$$

If $\omega / \Omega=m / n$, where $m$ and $n$ are integers, we can find a general expression for $\delta_{\text {cr }}$ so that each case of $m / n$ should be analyzed separately. It is clear, however, that commensurability of the frequencies lies between the cases " $\omega=\Omega$ " and " $m / n$ is irrational." Therefore, from (7) and (8) we get the critical value of $\delta_{\mathrm{cr}}$,

$$
\frac{3}{2 \kappa^{2}}|A-B|<\delta_{\mathrm{cr}}<\frac{3}{2 \kappa^{2}}(A+B) .
$$

For each $\delta_{\text {cr }}$ the region of chaos is then given by $\Delta_{c}$ $=\left[0, \delta_{\mathrm{cr}}\right]$.

We emphasize that $\Delta_{c}$ defines the value set $\delta$ for which homoclinic points exist. This is only a local criterion of the development of chaos in a small enough neighborhood of the unperturbed separatrix.

\section{A. Numerical analysis}

We numerically analyzed the Duffing-Holmes equation for the following fixed parameters: $\beta=8, \gamma=0.114, \eta=0.03$, $\omega=\Omega=1.1$, and $\delta$ is free. For these values, the expression (7) gives immediately $\delta_{\mathrm{cr}}=0.3798$. As a criterion of the chaoticity, we calculated the maximal Lyapunov exponent as a function of $\delta$. $^{23}$

A typical dependence of the maximal Lyapunov exponent on the parameter $\delta$ is shown in Fig. 2. Positive values mark clearly the region of chaos, $\Delta_{c}=[0.137,0.378]$. The same result takes place for any initial conditions chosen in a small separatrix neighborhood. The right boundary value of this region is in excellent agreement with $\delta_{\text {cr }}$ found analytically. On the contrary, the left boundary value of $\Delta_{c}$ cannot be obtained by the Melnikov method.

\section{TEMPORAL VARIATION OF THE PARAMETER $\delta$}

Having specified $\Delta_{c}$, let us introduce a parametric perturbation of $\delta$. We will show that for reasonable amplitudes of perturbation, the chaotic motion of the system (4) cannot be transformed into the regular oscillations. To be more precise, we consider the following equation:

$$
\begin{aligned}
& \dot{x}=y, \\
& \dot{y}=x-\beta(1+\eta \cos \Omega t) x^{3}-\left[\delta_{0}+\delta_{1} f(t)\right] y+\gamma \cos \omega t,
\end{aligned}
$$

where we made the replacement $\delta \rightarrow \delta(t)=\delta_{0}+\delta_{1} f(t)$ with a normalized periodical function $f(t)=f(t+T)$ and amplitude $\delta_{1}$. Let us further assume that perturbations should remain within the chaotic region, i.e., $\delta_{0} \in \Delta_{c}=\left[0, \delta_{\text {cr }}\right], \delta_{0}+\left|\delta_{1}\right|$ $<\delta_{\text {cr }}$, and $\delta_{0}-\left|\delta_{1}\right|>0$. Below we will show that in this case the system (10) does not possess regular dynamics.

We will use the Melnikov method, which gives conditions for the appearance of homoclinic points (and thus the chaos). Therefore, we will demand the absence of homoclinic points as a condition that the chaos is suppressed. The following numerical analysis will fortify our proceedings.

Let us assume that there exists a function $f(t)$ and an amplitude $\delta_{1}$ such that it stabilizes our system (10). Then $D\left(t_{0}\right)$ does not change its sign. We will show that this assumption cannot hold together with the requirement that the perturbation lies inside the chaotic region, i.e., $\delta(t) \in \Delta_{c}$ for all $t$.

\section{A. Harmonic perturbation}

In comparison with the analysis described in Sec. II, now $\delta$ has an additional term, $\delta_{1} f(t)$. Hence, we have to add to $D$ a new part $D_{1}\left(t_{0}\right): D\left(t_{0}\right)=D_{0}\left(t_{0}\right)+D_{1}\left(t_{0}\right)$. Here $D_{0}$ is the already known MF, $D_{0}\left(t_{0}\right)=-A \sin \omega t_{0}+B \sin \Omega t_{0}+C_{2}\left(\delta_{0}\right)$, and $D_{1}$ is determined by the additional part $\delta_{1} f(t)$ :

$$
D_{1}\left(t_{0}\right)=\int_{-\infty}^{+\infty} \delta_{1} f(t) y_{0}^{2}\left(t-t_{0}\right) d t .
$$

Because $\delta_{0} \in \Delta_{c}$, we know that $D_{0}\left(t_{0}\right)$ changes its sign. On the other hand, to eliminate chaos we should demand that $D\left(t_{0}\right)$ does not change its sign. Let us therefore consider the case that the sum $D_{0}\left(t_{0}\right)+D_{1}\left(t_{0}\right)$ has a non-negative value for all times $t_{0}$, i.e.,

$$
D_{1}\left(t_{0}\right) \geqslant-D_{0}\left(t_{0}\right) \text {. }
$$

In order to obtain $D_{1}$, we will analyze $f(t)$ in the form of a harmonic function $f(t)=\sin (\alpha t+\varphi)$. Then from (11) we have

$$
D_{1}\left(t_{0}\right)=\delta_{1} \kappa^{2} \nu_{\alpha} \sin \left(\alpha t_{0}+\varphi\right)
$$

with the constant $\nu_{\alpha}=\pi / 6 \alpha\left(2-\alpha^{2}\right) \operatorname{csch}(\pi \alpha / 2) \operatorname{sech}(\pi \alpha / 2)$. Thus, the expression (12) can now be rewritten as

$$
\delta_{1} \kappa^{2} \nu_{\alpha} \sin \left(\alpha t_{0}+\varphi\right) \geqslant A \sin \omega t_{0}-B \sin \Omega t_{0}-C_{2}
$$


Let us assume for a moment that $B=0$. This simplifies the last inequality containing three frequencies to an inequality with two frequencies. In this case, we can use the following suppression theorem: ${ }^{7}$

1. To satisfy inequality (14), it is necessary that $\alpha=p \omega$, where $p$ is an integer (resonance condition).

2. If additionally $p=(4 m+1-2 \varphi / \pi) /(4 n+1)$ for some integers $m$ and $n$, then (14) is satisfied only when

$$
\delta_{\min }<\delta_{1} \leqslant \delta_{\max }, \quad \delta_{\min }=\left(1-\frac{C_{2}}{A}\right) R,
$$

$\delta_{\max }=\frac{R}{p^{2}}$,

with $\quad R=3 \sqrt{2 \beta} \gamma / p\left(2-p^{2} \omega^{2}\right) \sinh (\pi p \omega / 2) / \cosh (\pi \omega / 2)$. (This holds only for $p \omega<\sqrt{2}$; at $p \omega>\sqrt{2}$ one has to change the roles of $\delta_{\min }$ and $\delta_{\max }$.)

This means that the value set $\delta_{1}$ leading to the chaos suppression crucially depends on the order of the resonance $p$. Now, it is necessary to estimate to what extent we can use the resonant perturbations of different order. To clarify this, let us consider what consequences are implied by the requirement $\delta_{\min }<\delta_{\max }$. Easy transformations result in

$$
\delta_{0}>\left(1-\frac{1}{p^{2}}\right) \delta_{\mathrm{cr}} \text {. }
$$

Because $\delta_{0}<\delta_{\text {cr }}$, for higher harmonics $(p>1)$ this inequality shrinks drastically the region of perturbations. It is evident that it remains valid if in (14) we take $B \neq 0$. That is why we confine ourselves by the first-order resonance $p=1$. In other words, following the suppression theorem, ${ }^{7}$ it is necessary to use perturbation of the form $\sin (p \omega+\phi)$. On the other hand (in agreement with the Melnikov approach), if $p>1$ we can apply such a perturbation only in a very small region on the edge of the chaotic region, i.e., only when $\delta_{0} \sim \delta_{\mathrm{cr}}$. Therefore, for an arbitrary $\delta_{0}$ in the region of chaos $\left[0, \delta_{\mathrm{cr}}\right]$ we have to take $p=1$. Then inequality (16) gives $0<\delta_{0}<\delta_{\text {cr }}$, which yields the whole chaos region $\Delta_{c}$.

The optimal value of the initial phase $\varphi$ for suppressing chaos is $\varphi=0$. This has to be understood in the sense that this value allows the smallest amplitudes of the chaossuppressing perturbation. Thus, together with the resonance condition $(\alpha=\Omega=\omega)$, from (14) we get

$$
\left(C_{1}+\delta_{1} \kappa^{2} \nu\right) \sin \omega t_{0}+C_{2} \geqslant 0,
$$

where $C_{1}=B-A, C_{2}=2 \kappa^{2} \delta_{0} / 3, \nu=\left.\nu_{\alpha}\right|_{\alpha=\omega}$. One can show that this inequality is equivalent to the following:

$$
\left|\delta_{1}+\frac{C_{1}}{\kappa^{2} \nu}\right| \leqslant \frac{2}{3|\nu|} \delta_{0} .
$$

Then the solution set for $\delta_{1}$ turns out to be directly visible. In Fig. 3 the set $S_{1}$ of pairs $\left(\delta_{0}, \delta_{1}\right)$ for which the chaotic motion vanishes [i.e., $D\left(t_{0}\right)$ does not change its sign] is shown.

However, we consider perturbations of $\delta$ that should remain within the chaotic region $\left[0, \delta_{\mathrm{cr}}\right]$. Therefore, $\delta_{0}+\left|\delta_{1}\right|$ $\leqslant \delta_{\text {cr }}=3 / 2 \kappa^{2}\left|C_{1}\right|$ or

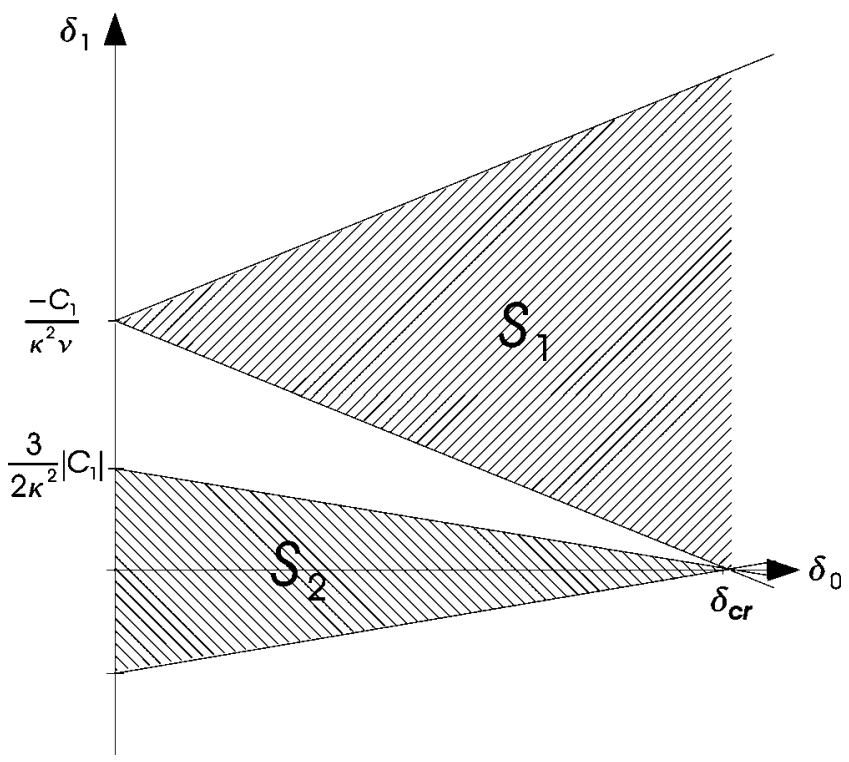

FIG. 3. The set $S_{1}$, where we have the stabilizing of chaotic dynamics, and the set $S_{2}$ of pairs $\left(\delta_{0}, \delta_{1}\right)$, satisfying the condition $\delta_{0}+\left|\delta_{1}\right|<\delta_{\text {cr }}$. One can see that these regions are disjoint.

$$
\left|\delta_{1}\right|<\frac{3}{2 \kappa^{2}}\left|C_{1}\right|-\delta_{0} .
$$

Thus, this inequality induces a set $S_{2}$ of "allowed" pairs $\left(\delta_{0}, \delta_{1}\right)$.

The set $S_{2}$ is also shown in Fig. 3. From this figure we can clearly see that the regions $S_{1}$ and $S_{2}$ are not intersecting with each other. This means that there are no pairs $\left(\delta_{0}, \delta_{1}\right)$ of perturbations within the region $\Delta_{c}$, satisfying the stabilizing condition.

Let us therefore show that for any $\left(\delta_{0}^{*}, \delta_{1}^{(1)}\right) \in S_{1}$ and $\left(\delta_{0}^{*}, \delta_{1}^{(2)}\right) \in S_{2}$ we have $\left|\delta_{1}^{(1)}\right|>\left|\delta_{1}^{(2)}\right|$. To make this, it is sufficient to show that at $\delta_{0}^{*}=0$ the maximal possible value of $\left|\delta_{1}^{(2)}\right|$ is less than $\left|\delta_{1}^{(1)}\right|$ and that at $\delta_{0}^{*}=\delta_{c}$ the minimal possible value of $\left|\delta_{1}^{(1)}\right|$ (as well as $\left|\delta_{1}^{(1)}\right|$ ) is equal to zero.

At $\delta_{0}^{*}=0$ [see (19)] we get $\left|\delta_{1}^{(2)}\right|=3 / 2 \kappa^{2}\left|C_{1}\right|$. On the other hand, from (18), $\left|\delta_{1}^{(1)}\right|>3 / 2 \kappa^{2}\left|C_{1}\right|=\left|\delta_{1}^{(2)}\right|$ because

$$
\max _{\alpha>0} \nu_{\alpha}<\frac{2}{3} \text {. }
$$

This last fact is obtained from the analysis $\nu_{\alpha}=\pi / 6 \alpha(2$ $\left.-\alpha^{2}\right) \operatorname{csch}(\pi \alpha / 2) \operatorname{sech}(\pi \alpha / 2)$. Thus, the maximal value is $2 / 3$ at $\alpha=0$.

Consider now some $\left|\delta_{1}^{(1)}\right| \in S_{1}$ and $\left|\delta_{1}^{(2)}\right| \in S_{2}$ at $\delta_{0}^{*}=\delta_{\mathrm{cr}}$ $=3 / 2 \kappa^{2}\left|C_{1}\right|$. It is obvious that $\delta_{1}^{(2)}=0$. According to inequality (18) at $\delta_{0}^{*}=\delta_{\mathrm{cr}}$ the boundaries of $S_{1}$ are determined as follows:

$$
\left|\delta_{1}^{(1)}+\frac{C_{1}}{\kappa^{2} \nu}\right|=\frac{2}{3|\nu|} \delta_{\mathrm{cr}}=\left|\frac{C_{1}}{\kappa^{2} \nu}\right| .
$$

Therefore, the minimal value of $\left|\delta_{1}^{(1)}\right|$ is also equal to zero.

Thus, Fig. 3 correctly illustrates the relation of $S_{1}$ and $S_{2}$, i.e., $S_{1} \cap S_{2}=\varnothing$.

It should be noted that in order to find the regular dynamics, we have analyzed the inequality $D\left(t_{0}\right) \geqslant 0$ for all $t_{0}$. Following to the Melnikov method, however, it is sufficient 


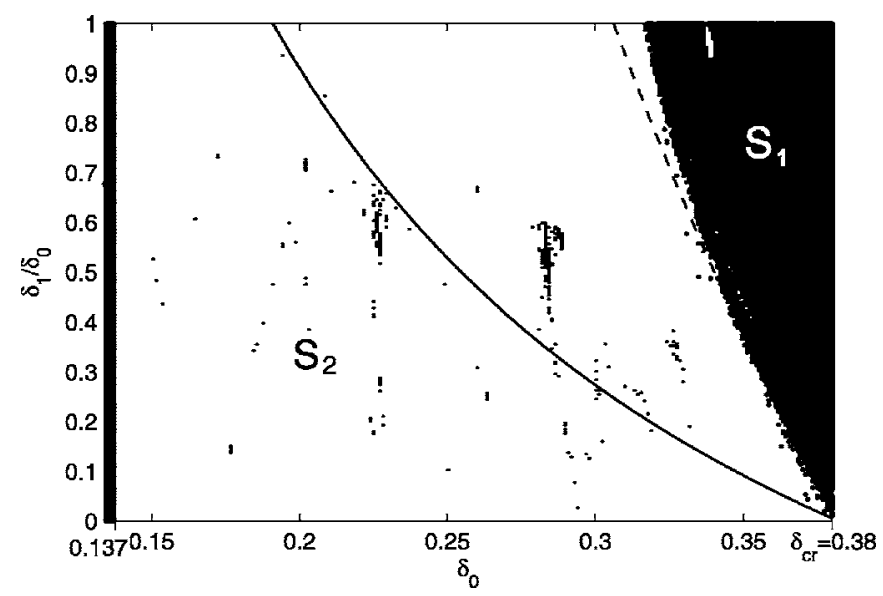

FIG. 4. The maximal Lyapunov exponent for the system (10). Each negative value is marked with a black dot. The solid line marks the upper boundary of $S_{2}$. The dashed line represents the boundary of $S_{1}$ obtained by the Melnikov method. The parameter values are the following: $\omega=\Omega=1.1, \gamma=0.114$, $\eta=0.03, \beta=8$. Initial conditions: $x_{0}=0, y_{0}=0$.

that $D\left(t_{0}\right)$ does not change its sign, which obviously also includes the case $D\left(t_{0}\right) \leqslant 0$ for all times $t_{0}$. Then the relation (17) may be changed by $\left(C_{1}+\delta_{1} \kappa^{2} \nu\right) \sin \omega t_{0}+C_{2} \leqslant 0$. But, because $C_{2}$ is a positive value, this inequality cannot be carried out.

Thus, we come to the conclusion that in the DuffingHolmes system the suppression of chaos cannot be achieved by a harmonic perturbation of the parameter $\delta$ within the region $\Delta_{c}$.

\section{Numerical analysis}

To analyze the perturbed system numerically, we computed the maximal Lyapunov exponent for different pairs $\left(\delta_{0}, \delta_{1}\right)$. For convenience, we have to norm $\delta_{1}$ to $\delta_{1} / \delta_{0}$. The result of $250 \times 250$ calculations of $\left(\delta_{0}, \delta_{1} / \delta_{0}\right), \delta_{0} \in \Delta_{c}$, is shown in Fig. 4.

One can see that our theoretical predictions are in fine agreement with the numerical analysis. In the region $S_{2}$ (the solid line) we have everywhere a positive value of the Lyapunov exponent. Therefore, here the perturbations are not stabilizing for the system. In the region $S_{1}$ (the dashed line), which was obtained by the Melnikov method, we clearly see the stabilization phenomenon.

\section{B. Perturbations with frequencies $\omega \neq \Omega$}

In the previous section a parametric perturbation with only one frequency was analyzed. As a consequence, to eliminate homoclinic points we had to put $\omega=\Omega$. Let us now suppose that $\omega \neq \Omega$. (The subharmonic resonances are not considered for the same reason as in Sec. III A: If we allowed higher-order resonances, the chaos suppression would not be possible in almost the whole region of chaos of the parameter $\delta_{0}$. Only in a very small region near $\delta_{\text {cr }}$ could one satisfy the suppression theorem. This does not yet mean that there exist chaos suppression perturbations that are strictly in the region of chaos.) In this case, it is necessary to vary $f(t)$, which induces (see the previous section) an additional summand $D_{1}\left(t_{0}\right)$ in the MF. We will require again that by this part the Melnikov function $D\left(t_{0}\right)$ does not change its sign. In particular, analogously to (12),

$$
D_{1}\left(t_{0}\right) \geqslant-D_{0}\left(t_{0}\right)=A \sin \omega t_{0}-B \sin \Omega t_{0}-C_{2}
$$

for all $t_{0}$, where $D_{0}\left(t_{0}\right)$ changes sign in the analyzed chaotic region. If we arrange $f(t)$ as a sum of harmonic functions with different frequencies, then, according to (13), we obtain that the left-hand side of the last inequality can also be represented by a sum of harmonic functions with the same frequencies.

According to the suppression theorem (see Sec. III A), inequality (21) can be satisfied if both sides consist of the same frequencies considered as before only in the first-order resonance. That is why we should take the perturbation as follows:

$$
f(t)=a_{1} \sin \omega t+a_{2} \sin \Omega t,
$$

where $a_{1}$ and $a_{2}$ are coefficients satisfying the relation

$$
\left|a_{1}\right|+\left|a_{2}\right|=1 \text {. }
$$

Thus, the correcting term in the $\mathrm{MF}$ is $D_{1}\left(t_{0}\right)$ $=\delta_{1} \kappa^{2}\left(a_{1} \nu_{\omega} \sin \omega t_{0}+a_{2} \nu_{\Omega} \sin \Omega t_{0}\right)$. Therefore, inequality (21) yields

$\left(\delta_{1} \kappa^{2} a_{1} \nu_{\omega}-A\right) \sin \omega t_{0}+\left(\delta_{1} \kappa^{2} a_{2} \nu_{\Omega}+B\right) \sin \Omega t_{0}+C_{2} \geqslant 0$.

The chaos suppression phenomenon is equivalent to the satisfaction of this inequality for some $a_{1}, a_{2}$, and $\delta_{1}>0$. Because the factors before sin should be as small as possible (we remind the reader that $C_{2}>0$ ), without loss of generality we can require that $a_{1} \nu_{\omega}>0$ and $a_{2} \nu_{\Omega}<0$.

Furthermore, according to our strategy, perturbations should belong entirely to $\Delta_{c}$. This region can be exactly specified in the particular cases " $\omega=\Omega$ " and " $\omega / \Omega$ is irrational" [see (7) and (8)]. Hence, for perturbations with two (different) frequencies, the inequality (24) may be considered when $\omega$ and $\Omega$ are incommensurable. Below we will focus on this case.

First of all, note that the inequality (24) is equivalent to the following:

$$
\begin{aligned}
\min _{t_{0} \in \mathbf{R}}[ & \left.\left(\delta_{1} \kappa^{2} a_{1} \nu_{\omega}-A\right) \sin \omega t_{0}+\left(\delta_{1} \kappa^{2} a_{2} \nu_{\Omega}+B\right) \sin \Omega t_{0}\right] \\
+ & +C_{2} \geqslant 0 .
\end{aligned}
$$

Owing to the incommensurability of $\omega$ and $\Omega$, we can evaluate separately the minimal value of every summand. Thus, from (24) we have

$$
\left|\kappa^{2} a_{1} \nu_{\omega} \delta_{1}-A\right|+\left|\kappa^{2}\right| a_{2} \nu_{\Omega}\left|\delta_{1}-B\right| \leqslant C_{2} .
$$

This inequality can be easily analyzed geometrically (Fig. 5).

In this figure, both summands of inequality (25) are shown by thin lines, and the total sum on the left-hand side is shown by the bold line. Considering the right-hand side, $C_{2} \propto \delta_{0}$, we can find the set $S_{1}$ of inequality (25). Because this inequality is symmetrical with respect to both summands, without loss of generality we can assume that the zero of the second summand lies to the left of the zero of the first summand: $B /\left(\kappa^{2}\left|a_{2} \nu_{\Omega}\right|\right)<A /\left(\kappa^{2} a_{1} \nu_{\omega}\right)$. 


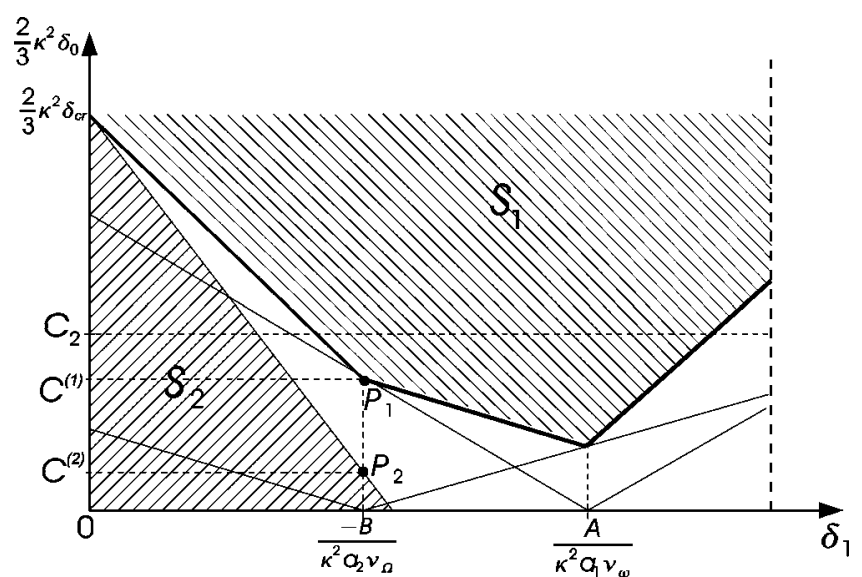

FIG. 5. The set $S_{1}$ of the stabilizing values $\delta_{1}$ [see inequality (25)] for the given $C_{2} \propto \delta_{0}$ and the set $S_{2}$ satisfying the condition that perturbations should belong to the chaotic region $\Delta_{\mathrm{cr}}$

Now let us define $S_{2}$ as the set of pairs $\left(\delta_{0}, \delta_{1}\right)$ for which the perturbation remains within the chaotic region $\Delta_{c}: \delta_{0}$ $+\left|\delta_{1}\right| \leqslant \delta_{\text {cr. }}$. Then we can show that $S_{1}$ and $S_{2}$ are not intersecting with each other, which means that perturbations inside $\Delta_{c}$ cannot be found.

Consider the values $C^{(1)}$ and $C^{(2)}$, which are the $y$ coordinate of the points $P_{1}$ and $P_{2}$, respectively (see Fig. 5). To find that $S_{1} \cap S_{2}$ is the empty set, it is sufficient to obtain that $C^{(1)}>C^{(2)}$, and both the upper bound of $S_{2}$ and the bold line [i.e., the left-hand side of inequality (25)] meet at the point $\delta_{1}=0$. The last condition is obvious. Thus, we should show that $C^{(1)}>C^{(2)}$.

The explicit forms of $C^{(1)}$ and $C^{(2)}$ are

$$
\begin{aligned}
& C^{(1)}=\left|\kappa^{2} a_{1} \nu_{\omega} \frac{-B}{\kappa^{2} a_{2} \nu_{\Omega}}-A\right|=\left|\frac{a_{1} \nu_{\omega}}{a_{2} \nu_{\Omega}} B+A\right|, \\
& C^{(2)}=\frac{2}{3} \kappa^{2} \delta_{0}=\frac{2}{3} \kappa^{2}\left(\delta_{\mathrm{cr}}-\delta_{1}\right)=A+B+\frac{2}{3} \frac{B}{a_{2} \nu_{\Omega}} .
\end{aligned}
$$

Thus, it is necessary for the inequality

$$
A+B+\frac{2}{3} \frac{B}{a_{2} \nu_{\Omega}}<\left|\frac{a_{1} \nu_{\omega}}{a_{2} \nu_{\Omega}} B+A\right|
$$

to be satisfied. First, consider the case when the expression under the module sign is not negative. Then inequality (26) can be rewritten as $-\left|a_{1} \nu_{\omega}\right|-\left|a_{2} \nu_{\Omega}\right|+2 / 3>0$. In fact, this is correct because $-\left|a_{1} \nu_{\omega}\right|-\left|a_{2} \nu_{\Omega}\right|+2 / 3>-2 / 3\left(\left|a_{1}\right|+\left|a_{2}\right|\right)$ $+2 / 3=0$. Here we have used the relations (20) and (23).

Let us now consider the case in which the expression under the module sign is negative, i.e., $A / B<a_{1} \nu_{\omega} /\left|a_{2} \nu_{\Omega}\right|$. Then inequality (26) is equivalent to $a_{1} \nu_{\omega}-(2 A / B$ $+1)\left|a_{2} \nu_{\Omega}\right|+2 / 3>0$. This inequality is also right because

$$
\begin{aligned}
a_{1} \nu_{\omega} & -\left(2 \frac{A}{B}+1\right)\left|a_{2} \nu_{\Omega}\right|+\frac{2}{3} \\
> & a_{1} \nu_{\omega}-\left(2 \frac{a_{1} \nu_{\omega}}{\left|a_{2} \nu_{\Omega}\right|}+1\right)\left|a_{2} \nu_{\Omega}\right|+\frac{2}{3} \\
& =-a_{1} \nu_{\omega}-\left|a_{2} \nu_{\Omega}\right|+\frac{2}{3} \\
> & -\frac{2}{3}\left(\left|a_{1}\right|+\left|a_{2}\right|\right)+\frac{2}{3}=0 .
\end{aligned}
$$

\section{CONCLUSION}

The development of the theory of dynamical systems and numerous studies of nonlinear processes has shown that chaotic behavior is typical and prevalent in many nonlinear processes. Nowadays it is obvious that chaotic properties are inherent in the overwhelming majority of systems, and if chaos is not detected, this is perhaps due to the fact that it arises in very small regions of the parametric space or for nonphysical parameter values. The predictability problem, which first arose for fairly complex systems (such as hydrodynamic systems or systems in statistical mechanics), became common for many fields of modern science.

At the same time, as is known, chaotic dynamical systems are pliable to external perturbations. This property can be used to the control of dynamical systems and suppression of the onset of the undesirable chaotic regime. This means that parametric perturbations should be determined at the set of their values for which the system has a positive maximal Lyapunov exponent. If this is not the case, we cannot assess the chaos suppression. This is the main sense of this phenomenon. However, in the present article we come to the main conclusion that the phenomenon of chaos suppression cannot be achieved via arbitrary parametric excitations.

Analyzing the known Duffing-Holmes model (1), we found that chaotic dynamics cannot be suppressed by a harmonic perturbation of the parameter $\delta$, independently from the other parameter values. In addition, we have shown in the case in which $\omega$ and $\Omega$ are incommensurable that chaos suppression cannot be realized even by an arbitrary periodic perturbation of $\delta$. On the basis of our analysis, we have come to the conclusion that to stabilize the behavior of a chaotic system, the perturbation and parameters must be carefully chosen. This contradicts many previous expectations that the chaos may always be suppressed parametrically. If, however, we apply short-time impulse perturbations of the parameter $\delta$, then we can easily stabilize the system dynamics. ${ }^{24}$ One can expect that similar results will be observed in other systems.

In this connection, it would be useful to have a basic criterion that would allow us to determine in what cases the chaotic motion can be stabilized by parametric excitations. This important question will arise every time in practice. However, as of yet there are not even general analytical criteria of the existence of chaos in quite simple dynamical systems. 
${ }^{1}$ V. V. Alekseev and A. Loskutov, Moscow Univ. Phys. Bull. 40, 46 (1985).

${ }^{2}$ V. V. Alexeev and A. Loskutov, Sov. Phys. Dokl. 32, 270 (1987).

${ }^{3}$ R. Lima and M. Pettini, Phys. Rev. A 41, 726 (1990).

${ }^{4}$ G. Cicogna and L. Fronzoni, Phys. Rev. E 47, 4585 (1993).

${ }^{5}$ A. Loskutov and A. I. Shishmarev, Russ. Math. Surveys 48, 183 (1993).

${ }^{6}$ A. Loskutov and A. I. Shishmarev, Chaos 4, 351 (1994).

${ }^{7}$ R. Chacón, Phys. Rev. E 51, 761 (1995).

${ }^{8}$ R. Chacón, Phys. Rev. Lett. 86, 1737 (2001).

${ }^{9}$ Y. Braiman and I. Golhirsh, Phys. Rev. Lett. 66, 2545 (1991).

${ }^{10}$ A. Loskutov, Comput. Math. and Modeling 12, 314 (2001).

${ }^{11}$ E. Ott, C. Grebogi, and J. A. Yorke, Phys. Rev. Lett. 64, 1196 (1990).

${ }^{12}$ T. Shinbrot, C. Grebogi, E. Ott, and J. A. Yorke, Nature (London) 363, 411 (1993).

${ }^{13}$ S. Boccaletti, C. Grebogi, Y.-C. Lai et al., Phys. Rep. 329, 103 (2000).

${ }^{14}$ J. Guckenheimer and P. Holmes, Nonlinear Oscillations, Dynamical Sys- tems, and Bifurcations of Vector Fields (Springer-Verlag, New York, 1990).

${ }^{15}$ Yu. I. Neimark and P. S. Landa, Stochastic and Chaotic Oscillations (Kluwer Academic, Dordrecht, 1987).

${ }^{16}$ R. Lima and M. Pettini, Int. J. Bifurcation Chaos Appl. Sci. Eng. 8, 1675 (1998).

${ }^{17}$ V. K. Mel'nikov, Trans. Mosc. Math. Soc. 12, 1 (1963)

${ }^{18}$ A. J. Lichtenberg and M. A. Lieberman, Regular and Stochastic Motion (Springer-Verlag, 1990).

${ }^{19}$ Y. Kivshar, F. Rodelsperger, and H. Benner, Phys. Rev. E 49, 319 (1994).

${ }^{20}$ L. Fronzoni and M. Giocondo, Int. J. Bifurcation Chaos Appl. Sci. Eng. 8, 1693 (1998).

${ }^{21}$ P. J. Holmes and F. C. Moon, ASME J. Appl. Mech. 50, 1021 (1983).

${ }^{22}$ F. Cuadros and R. Chacón, Phys. Rev. E 47, 4628 (1993).

${ }^{23}$ G. Benettin, L. Galgani, and J. M. Strelcyn, Phys. Rev. A 14, 2338 (1976)

${ }^{24}$ A. Loskutov and A. R. Dzhanoev, J. Exp. Theor. Phys. 98, 1045 (2004). 